\title{
LiC $_{6}$ Heterogeneous Interface for Stable Lithium Plating and Stripping
}

\author{
Zibo Zhang ${ }^{1,2}$, Wei Deng ${ }^{1}$, Xufeng Zhou ${ }^{1,2, *}$, Zhaoping Liu ${ }^{1,2,{ }^{*}}$ \\ ${ }^{1}$ Advanced Li-ion Battery Engineering Laboratory, CAS Engineering Laboratory for Graphene, Key Laboratory of Graphene \\ Technologies and Applications of Zhejiang Province, Ningbo Institute of Materials Technology and Engineering (NIMTE), \\ Chinese Academy of Sciences, Ningbo 315201, Zhejiang Province, China. \\ ${ }^{2}$ College of Materials Science and Opto-Electronic Technology, University of Chinese Academy of Sciences, Beijing 100049, China.
}

\begin{abstract}
Lithium metal has the highest theoretical specific energy density $\left(3860 \mathrm{mAh} \cdot \mathrm{g}^{-1}\right)$ and the most negative redox potential $(-3.04 \mathrm{~V}$ vs standard hydrogen electrode) among all alkali metals. These features have attracted the interest of battery researchers to put lithium metal into practical use in rechargeable batteries. However, lithium metal tends to deposit as dendritic or mossy morphology during the charging process, and such non-uniform deposition induces low Coulombic efficiency and poor cycling stability. In addition, dendritic metallic lithium can easily
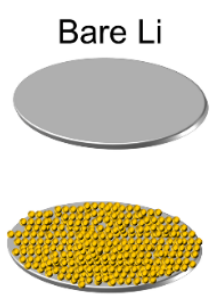
Li plating

Li@C penetrate the separator, which causes internal short circuit and leads to severe safety issues. Thus it is important to control the electrodeposition process of lithium to inhibit the formation of $\mathrm{Li}$ dendrites. Surface modification of lithium is a widely adopted strategy that can induce uniform deposition of Li. In this paper, a $\mathrm{LiC}_{6}$ heterogeneous interfacial layer is decorated on the surface of lithium metal anode. It is prepared in a simple manner by mechanically rolling graphitized carbon nanospheres on a $\mathrm{Li}$ foil. The increase in surface area by this $\mathrm{LiC}_{6}$ layer can homogenize the current density on the surface of the lithium foil. Simultaneously, the electronegativity of $\mathrm{LiC}_{6} \mathrm{can}_{\text {also }}$ homogenize the lithium ion flux. The effect of heterogeneous interface on the electrochemical plating and stripping behavior of lithium in carbonate electrolyte is also studied. Morphological characterization and electrochemical performance tests reveal that the $\mathrm{LiC}_{6}$ heterogeneous interface can significantly improve the reversibility and uniformity of the electrochemical plating and stripping of $\mathrm{Li}$, thereby inhibiting dendritic growth and maintaining the stability of the anode/electrolyte interface. Alternating current electrochemical impedance spectroscopy analysis determines that the solid electrolyte interface (SEI) impedance of bare lithium decreases from the initial 275 to $100 \Omega$ as the deposition capacity increases, suggesting that severe rupture of the SEl is caused by the huge volume change after lithium deposition. On the contrary, the SEl impedance of the lithium foil with the $\mathrm{LiC}_{6}$ heterogeneous interface layer remains nearly constant (from the initial 26 to $25 \Omega$ after electrodeposition) indicates that the $\mathrm{LiC}_{6}$ layer is able to inhibit dendrite growth and stabilize the interface. Thus, stable lithium plating/stripping over $300 \mathrm{~h}$ is achieved at a current density of $1 \mathrm{~mA} \cdot \mathrm{cm}^{-2}$ and at a fixed capacity of $1 \mathrm{mAh} \cdot \mathrm{cm}^{-2}$ with a voltage hysteresis of less than $50 \mathrm{mV}$. The $\mathrm{Li}_{-}-\mathrm{LiFePO}_{4}$ full cell test demonstrates that the cycling stability of the modified lithium metal anode is superior to that of the bare one.
\end{abstract}

Key Words: Lithium metal secondary battery; Lithium metal anode; Dendrite; Heterogeneous interface; LiC 6

Received: August 25, 2020; Revised: September 27, 2020; Accepted: October 6, 2020; Published online: October 22, 2020.

${ }^{*}$ Corresponding authors. Emails: zhouxf@nimte.ac.cn (X.Z.). liuzp@nimte.ac.cn (Z.L.).

(C) Editorial office of Acta Physico-Chimica Sinica 


\title{
稳定锂电化学沉积和溶解行为的 $\mathrm{LiC}_{6}$ 异质微结构界面层
}

\author{
张自博 1,2 , 邓伟 1 , 周旭峰 $1,2,{ }^{*}$, 刘兆平 $1,2,{ }^{*}$ \\ 1 中国科学院宁波材料技术与工程研究所, 动力锂电池工程实验室, 中国科学院石墨烯工程实验室, 浙江省石墨烯应用研究 \\ 重点实验室, 浙江 宁波 315201 \\ 2 中国科学院大学材料科学与光电技术学院, 北京 100049
}

\begin{abstract}
摘要: 本文采用机械辊压方法在金属锂表面通过原位固相反应生成 $\mathrm{LiC}_{6}$ 异质微结构界面层, 并研究了在碳酸酯有机电解 液体系下该异质层对锂电化学沉积和溶解行为的影响。通过形貌表征与电化学测试发现, $\mathrm{LiC}_{6}$ 异质层能够有效提升锂电 化学沉积的可逆性与均匀性, 从而抑制枝晶生长及维持沉积/溶解界面的稳定。使用异质层改性金属锂负极的扣式全电池 也较纯金属锂负极体系表现出更为优异的循环稳定性。
\end{abstract}

关键词：锂金属二次电池；金属锂负极；枝晶；异质微结构； $\mathrm{LiC}_{6}$ 中图分类号: 0646

\section{1 引言}

金属锂具有极高的理论比容量 (3860 $\mathrm{mAh} \cdot \mathrm{g}^{-1}$ )和最低的氧化还原电位 $(-3.04 \mathrm{~V} v s$. SHE (standard hydrogen electrode)), 因此对于可充电二 次电池而言是一种十分理想的负极材料, 在过去 的 40 多年中一直成为研究热点 ${ }^{1-4}$ 。然而金属锂负 极的问题也十分突出, 阻碍了锂金属二次电池的 商业化应用。金属锂负极的主要问题包括在充放 电过程中锂枝晶的生长, 以及固态电解质膜(SEI) 的不断破裂和再生, 从而造成库伦效率降低与电 解液的持续损耗 $5-7$ 。不可控的枝晶生长最终又会 造成电池短路, 从而引发热失控。锂枝晶生长主要 是由于金属锂表面的不均匀性及锂的高反应活性 导致的锂离子的不均匀形核所产生的 8,9 。金属锂 沉积涉及电子和离子两个要素, 电流密度和锂离 子通量会极大影响锂离子沉积的均匀性和致密 性, 因此如何有效调控沉积界面处的电子与离子 状态是抑制锂枝晶生长的重要策略, 而表面功能 层的构建是实现这一策略的重要手段。例如, 从调 控锂离子通量角度考虑, 有研究通过涂敷转移的 方法在锂䇴表面修饰一层锂离子导体材料 $\mathrm{Li}_{2} \mathrm{TiO}_{3}$, 以此来加速和均化锂离子通量, 从而实 现了锂的均匀沉积 ${ }^{10}$ 。Lee等 ${ }^{11}$ 则通过在锂馢表面 转移一层 $\mathrm{Cu}_{3} \mathrm{~N}$ 纳米线来调控锂离子通量, 有效地 抑制了锂枝晶的生长, 提高了电池的循环寿命和库 伦效率。从分散表面电流密度角度考虑, Qian等 ${ }^{12}$ 通 过原位化学反应在锂䈃表面生成一层纳米多孔铜 颗粒层, 通过对电流密度的均匀分散实现锂离子 的均匀沉积, 有效抑制了枝晶状锂的产生, 提高了 电池的循环寿命。上述策略起到了稳定锂的沉积 和溶解行为的作用, 但在改性材料的易得性与表
面修饰的操作便利性等方面仍存在不足。

结构形态丰富, 且简便易得的碳基材料被证 明可以有效改善锂沉积/溶解过程的可逆性与稳定 性 ${ }^{13-21}$ 。在各类碳材料中, 石墨化碳在热力学上能 与金属锂发生自发反应 ${ }^{22}$ 。石墨锂化后(最终形成 $\mathrm{LiC}_{6}$ ), 由于锂把电子转移给碳, 因此具有较强的 电负性, 能够与电解液中的锂离子产生较强的相 互作用以调控其扩散行为, 而且在室温下, 锂离子 和电子可以自发地在锂化石墨层中扩散 ${ }^{23}, \mathrm{LiC}_{6}$ 离 子电导率可以达到 $5.7 \times 10^{-5} \mathrm{~S} \cdot \mathrm{cm}^{-1} 24$ 。因此, 通 过锂化石墨改性有助于引导锂的均匀沉积 25 。然 而, 传统石墨类材料由于其颗粒尺寸大, 从动力学 角度不利于锂插层反应的发生, 因此难以在温和 条件下生成锂化石墨。

本文通过简单的机械辊压方法将具有超小粒 径的石墨化碳纳米球均匀附着于锂䈃表面, 生成 一层 $\mathrm{LiC}_{6}$ 异质微结构界面层, 起到均化锂箔表面 电流密度和锂离子通量的目的, 从而有效提升了 锂沉积与溶解行为的均匀性与可逆性。

\section{2 实验部分}

\section{1 原材料与试剂}

碳纳米球购自纳富迪斯科技公司 (Nafortis Technology); 锂䇴购自天津中能锂业; 电解液为 1 $\mathrm{mol} \cdot \mathrm{L}^{-1} \mathrm{LiPF}_{6}$ 的碳酸乙烯酯 $(\mathrm{EC}) /$ 碳酸二乙酯 (DEC) $(V / V=1: 1)$ 溶液。隔膜采用Celgard 2400。

\section{2 材料制备}

将商业化的碳纳米球在 $100{ }^{\circ} \mathrm{C}$ 真空烘箱中干 燥 $12 \mathrm{~h}$, 然后在 $\mathrm{H}_{2} \mathrm{O}$ 和 $\mathrm{O}_{2}$ 含量均低于 $0.1 \times 10^{-6}$ 的 $\mathrm{Ar}$ 保护的手套箱中, 将 $2 \mathrm{mg}$ 碳纳米球通过简单的辊 压操作负载在 $2 \mathrm{~cm}^{2}$ 的新鲜金属锂箔表面, 待金属 
锂表面迅速转变为金黄色后, 即制备出 $\mathrm{LiC}_{6}$ 异质 微结构界面层修饰的锂箔材料 $(\mathrm{Li} @ \mathrm{C})$ 。

\section{3 电池组装}

通过组装成CR2032型纽扣电池进行电化学 性能测试。恒电流充放电测试采用对称型电池, 以 $\mathrm{Li} @ \mathrm{C}$ 为正负极, 以 Celgard 2400 为隔膜, 使用 1 $\mathrm{mol} \cdot \mathrm{L}^{-1} \mathrm{LiPF}_{6} \mathrm{EC} / \mathrm{DEC}(V / V=1: 1)$ 的有机溶液为 电解液, 而对比电池采用未改性的金属锂䈃为正 负极。全电池电化学性能测试中, 正极以磷酸铁锂 (LFP)为活性物质, Super P为导电剂, 聚偏氟乙烯 (PVDF)为粘结剂, 三者按质量比 $8: 1: 1$ 混合在 $N$ 甲基吡咯烷酮(NMP)溶剂中均匀搅拌, 随后用刮刀 涂敷在铝簿上, $80{ }^{\circ} \mathrm{C}$ 真空干燥 $10 \mathrm{~h}$ 后裁成直径为 $13 \mathrm{~mm}$ 的极片 (活性物质载量为 $5 \mathrm{mg} \cdot \mathrm{cm}^{-2}$ ), 负极分 别采用 $\mathrm{Li} @ \mathrm{C}$ 和未改性的 $\mathrm{Li}$ 为对比电极, 隔膜同样 采用Celgard 2400, 电解液与对称电池采用的电解 液相同。循环后的锂箔用碳酸二甲酯(DMC)溶剂 清洗除去表面杂质, 在手套箱中自然干燥 $24 \mathrm{~h}$, 然 后快速转移到扫描电子显微镜中进行形貌表征。

\section{4 材料结构表征与电化学性能测试}

用D8型粉末 X射线衍射仪(XRD, AXS)和共聚 焦拉曼光谱仪(Renishaw inVia Reflex)测定材料的 结构。材料的微观形貌使用Hitachi S-48000冷场扫 描电子显微镜(SEM, FEI, Sirion 200)和透射电子 显微镜(TF20)进行分析。采用LAND CT2001A电池 测试设备进行电化学性能测试。恒电流充放电测 试的电流密度为 $1 \mathrm{~mA} \cdot \mathrm{cm}^{-2}$, 面积容量为 1 $\mathrm{mAh} \cdot \mathrm{cm}^{-2}$ 。扣式全电池测试的截止电压设置为 2.8-4 V。电化学阻抗谱(Electrochemical impedance spectroscopy, EIS)的测试采用Solartron 1400型电 化学工作站, 测试的频率范围为 $1 \mathrm{MHz}-100 \mathrm{mHz}$, 微扰动电压设置为 $5 \mathrm{mV}$ 。

\section{3 结果与讨论}

\section{1 异质微结构界面层制备及表征}

图1 a 是异质微结构界面层制备过程示意图, 将 适量具有石墨化结构和超小粒径 $(<50 \mathrm{~nm})$ 的碳纳 米球(图S1和S2, Supporting Information)放置在新 鲜的金属锂簿表面, 通过简单的机械辊压手段实 现金属锂与碳纳米球的复合。图 $1 \mathrm{~b}$ 是原始锂箔的 光学照片, 可以看出典型的金属光泽。图 $1 \mathrm{c}$ 是 $\mathrm{LiC}_{6}$ 异质微结构界面层修饰的锂箔的光学照片, 表面 呈现均匀的金黄色, 表明碳纳米球与金属锂之间不 仅仅是简单的物理复合, 而是发生了化学反应, 形 成了锂碳化合物, 并从其颜色可以判断生成了 $\mathrm{LiC}_{6}$ 。图 $\mathrm{S} 3$ 是未改性的金属锂的表面形貌, 可以看 出表面有不均匀的凸起和划痕。图1d是 $\mathrm{Li} @ \mathrm{C}$ 的表 面形貌，可以看出修饰后锂片表面由大量的碳纳 米球堆积构成, 颗粒之间存在堆积空隙, 可以同时 起到分散电流和为锂提供沉积空间的作用。图1e 是 $\mathrm{Li} @ \mathrm{C}$ 的截面形貌, 从中可以看出修饰后的锂馢 表面具有厚度约为 $3 \mu \mathrm{m}$ 的碳层, 且表面平整。用 $\mathrm{X}$ 射线光电子能谱(XPS)对未改性锂箔以及Li@C 表 面进行化学组成的对比分析(图1g)发现, 表面改性 后的锂的 $1 s$ 谱峰向更高结合能处位置移位 (55.7 $\mathrm{eV})$, 也进一步验证了金属锂与碳纳米球之间发生 了明显的化学作用, 生成了 $\mathrm{LiC}_{6}$ 。碳纳米球与金属 锂之间能迅速反应形成锂碳化合物主要得益于该 碳材料独特的纳米结构。从高分辨TEM (图1f)可以 看出该碳纳米球具有高度石墨化结构, 石墨化区 域内 $c$ 轴方向的层数普遍较少(基本在 20 层以下), 并且取向各异, 类似于弯曲折叠的多层石墨烯。这

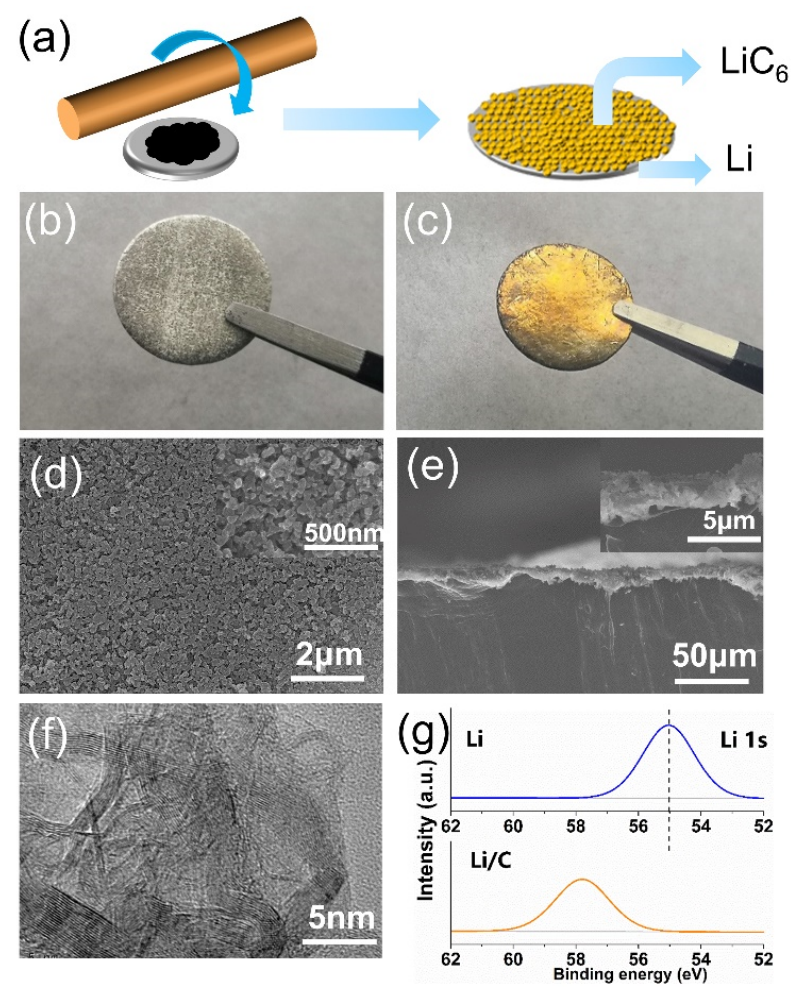

图1 (a)异质微结构界面层制备过程示意图; (b)锂簿的光 学照片; (c) Li@C的光学照片; (d) Li@C 表面SEM图;

(e) Li@C截面SEM图; (f)碳纳米球高分辨TEM图; (g) Li与 Li@C 的表面 Li 1s XPS谱图

Fig. 1 (a) Schematic diagram of the preparation process of the heterogeneous interface layer; (b) digital photograph of Li foil; (c) digital photograph of Li@C; (d) SEM image of Li@C surface; (e) cross-sectional SEM image of Li@C; (f) high resolution TEM image of carbon nanospheres; (g) Li 1s XPS spectra of Li and Li@C. 
一独特结构大幅改善了锂在石墨层间插层的动力 学过程, 从而在短时间内即可自发实现均匀彻底 的插层反应。作为对比, 我们又采用颗粒尺寸与形 貌类似, 但微观结构存在显出差异的导电碳黑 (Super P, 石墨化程度低)在锂箔表面进行了相同的 辊压处理, 结果发现使用普通碳黑处理的理管表 面始终保持黑色(图S4), 与碳纳米球修饰的锂䈃形 成鲜明对比, 进一步说明了高度石墨化碳纳米颗 粒是实现锂䈃表面 $\mathrm{LiC}_{6}$ 异质微结构改性的必要条 件。

\section{2 异质微结构界面层作用下的锂沉积行为}

为了探究修饰 $\mathrm{LiC}_{6}$ 异质微结构界面层的 $\mathrm{Li} @ \mathrm{C}$ 电极的沉积行为, 我们通过组装成对称电池 进行不同容量下的电化学沉积实验, 沉积的电化 学曲线如图S5 所示, 并对沉积后的Li@C电极进行 离位的 $\mathrm{SEM}$ 表征。当电沉积容量为 $0.1 \mathrm{mAh} \cdot \mathrm{cm}^{-2}$ 时, Li@C极片表面形貌与未沉积时类似, 依然可 以观察到比较明显的由纳米颗粒构成的异质微结 构(图2a)。当电化学沉积容量增加到 $0.5 \mathrm{mAh} \cdot \mathrm{cm}^{-2}$ 时, 可以观察到极片表面的三维结构变得不明显, 纳米颗粒间的孔隙逐渐被填充(图2b)。当电化学沉 积容量进一步增加至 $1 \mathrm{mAh} \cdot \mathrm{cm}^{-2}$ 时, 金属锂已经 完全覆盖了 $\mathrm{LiC}_{6}$ 异质层, 极片表面呈现光滑平整 的形貌(图2c)。该电极表面形貌的变化趋势表明金 属锂的沉积是一个自下而上逐步填充碳纳米球间 孔隙的过程。我们对未改性的锂箔也进行了相同 的电化学沉积实验, 当电沉积容量为 $0.1 \mathrm{mAh} \cdot \mathrm{cm}^{-2}$ 时, 锂䈃表面即出现不均匀的形核和生长(图2d)。 当沉积容量增加到 $0.5 \mathrm{mAh} \cdot \mathrm{cm}^{-2}$ 时, 锂箔表面出现 了典型的枝晶状结构(图2e), 并且锂枝晶会随着沉 积容量的增加进一步变得粗大(图2f)。两种负极上
锂沉积行为的显著差异表明, $\mathrm{LiC}_{6}$ 异质微结构层 能够有效引导锂离子的均匀沉积。碳纳米球与金 属锂之间自发的固相反应形成的 $\mathrm{LiC}_{6}$ 异质层具有 较强的电负性, 能够与电解液中的锂离子产生较 强的相互作用, 有助于均化锂离子的扩散行为, 从 而优化初次沉积过程中锂的形核, 使得后续沉积 更加均匀。而导电性优异的碳纳米球又能降低局 域电流密度, 也有助于锂的均匀沉积。此外, 由碳 纳米球构成的具有多孔结构的导电层又能为金属 锂沉积提供空间约束作用, 从而进一步改善了沉 积均匀性。

为了进一步探究 $\mathrm{LiC}_{6}$ 异质微结构界面层对锂 箔电极的影响, 我们采用交流阻抗法对未改性和 改性后的锂管在不同锂沉积容量下极片的界面情 况进行了分析。交流阻抗法的特点是可以在较小 的影响下精确测量锂箔的电化学特性和表界面特 性。图3a, b分别是未改性的锂箔和具有 $\mathrm{LiC}_{6}$ 异质 微结构界面层的锂簿在不同沉积容量下的Nyqusit 图, 从中可以发现两者在中频区的半圆均随着充 电量的增加而减小, 而未改性锂䇴的减小程度更 加显著。我们进而通过等效电路模拟来对 Nyquist 图进行定量分析。金属锂电极的界面可以等效为 $\mathrm{SEI}$ 膜的阻抗 $R_{f}$ 与电容 $C_{f}$ 并联, 进而与电解液本体 阻抗 $R_{\mathrm{S}}$ 串联(图 $\mathrm{S} 6$ )。从Nyquist图半圆直径可以求出 SEI电阻 $R_{f}$ 的数值, 膜电容可以根据公式 $C_{f}=$ $\left(2 \pi f \cdot R_{f}\right)^{-1}$ 求出, 其中 $f$ 为半圆最高点对应的频率 26 。 计算得到的阻抗与电容值如图 $3 \mathrm{c}$, d所示。对于未 改性锂箔, 随着沉积容量增加, 其 $R_{f}$ 值从初始的 275 $\Omega$ 迅速下降至 $1 \mathrm{mAh} \cdot \mathrm{cm}^{-2}$ 时的 $100 \Omega$, 同时 $C_{f}$ 也增 长了 $39 \%$, 说明在沉积过程中, 其界面性质发生了 显著性变化, 主要是由于锂枝晶的大量生长。与之
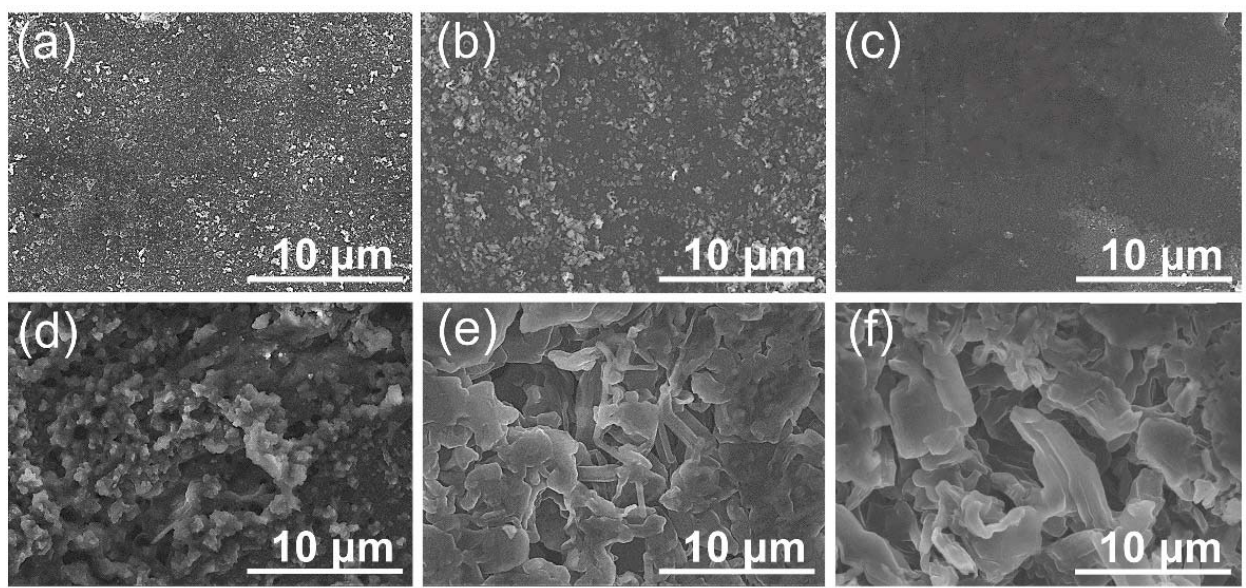

图2 电沉积 $0.1 、 0.5$ 和 $1 \mathrm{mAh} \cdot \mathrm{cm}^{-2}$ 三个不同面积容量时 $\operatorname{Li} @ C(a-c)$ 与纯锂馢 $(d-f)$ 表面的形貌变化

Fig. 2 Surface morphology of lithium deposited with different capacities of $0.1,0.5$ and $1 \mathrm{mAh} \cdot \mathrm{cm}^{-2}$ : 
(a)

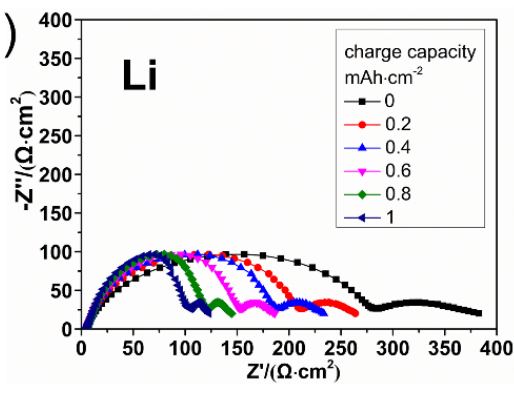

(c)

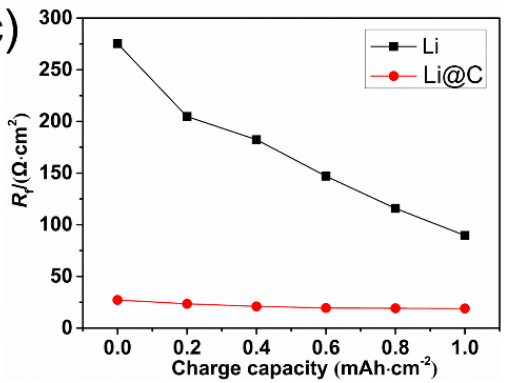

(b)

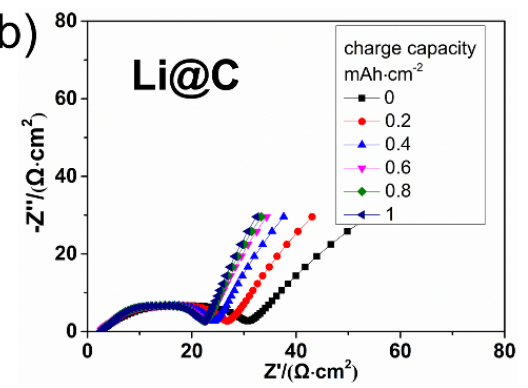

(d)

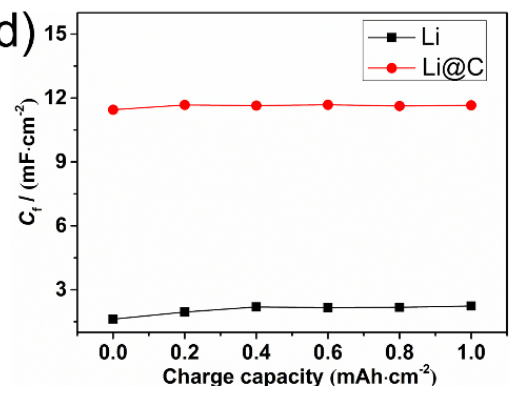

图3 (a-b)锂电极在不同沉积容量时的Nyquist图, (c-d) 电极充电时 $R_{f}$ 和 $C_{f}$ 随沉积容量的变化图

Fig. 3 (a-b) Nyquist diagram of lithium electrode at different charge capacity,

(c-d) plots of $R_{f}$ and $C_{f}$ values vs. charge capacity.

相比, $\mathrm{LiC}_{6}$ 异质微结构界面层改性锂箔的 $R_{f}$ 和 $C_{f}$ 在 沉积过程中几乎未发生变化, $R_{f}$ 仅从 $26 \Omega$ 减小至 25 $\Omega$, 说明其界面始终保持相对稳定的状态, 这与图 2 中观察到的均匀和致密的锂沉积形貌也形成了 很好的印证。

为了探究改性后锂箔的电化学性能, 把未处 理的锂䈃和改性后锂䈃分别组装成对称电池, 通 过恒电流方式进行充放电测试, 其结果如图4所 示。在电流密度为 $1 \mathrm{~mA} \cdot \mathrm{cm}^{-2}$ 、沉积容量为 1 $\mathrm{mAh} \cdot \mathrm{cm}^{-2}$ 的条件下, 未改性的锂箔在循环 30 圈后 (约 $60 \mathrm{~h}$ )即出现电压滞后的增大与波动, 这主要是 是由于电解液与金属锂的副反应产物以及死锂堆 积造成的界面阻抗增加所引起的。与之形成鲜明 对比的是, 具有异质微结构界面层的锂䇴在循环
$300 \mathrm{~h}$ 后仍保持较低的极化电压。虽然最终电压滞 后仍会增大，但相比未改性的锂䈃增加幅度明显 缩小, 说明均匀致密的锂沉积可以减小因低维度 锂枝晶的产生造成电解液加速分解的情况。为了 进一步说明异质层中 $\mathrm{LiC}_{6}$ 对于锂离子均匀沉积的 引导作用, 我们对导电碳黑(Super P)改性的锂簿进 行了同样的恒流充放电测试, 其结果如图S7所示。 碳黑修饰的锂箔可以稳定循环 $120 \mathrm{~h}$, 其后极化逐 渐增大, 虽优于没有修饰的锂箔, 但与具有 $\mathrm{LiC}_{6}$ 异 质微结构界面层的锂箔相比依然显示出明显劣 势。因此, 我们推测异质层中电负性的 $\mathrm{LiC}_{6}$ 对锂离 子的亲和性在锂的均匀沉积中起到了相当关键的 作用。

图5a, e分别是循环后未改性锂䇴和具有 $\mathrm{LiC}_{6}$

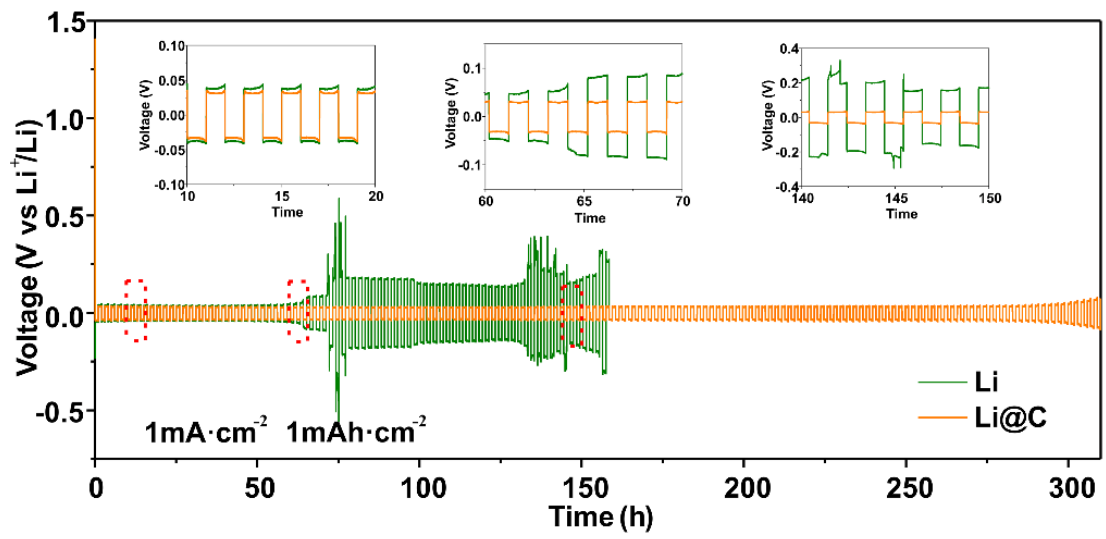

图4 以 $\mathrm{Li} @ \mathrm{C}$ 和纯锂簿为电极的对称电池的循环性能图

Fig. 4 Cycling performance of symmetric cells with $\mathrm{Li} @ \mathrm{C}$ and bare Li foil. 


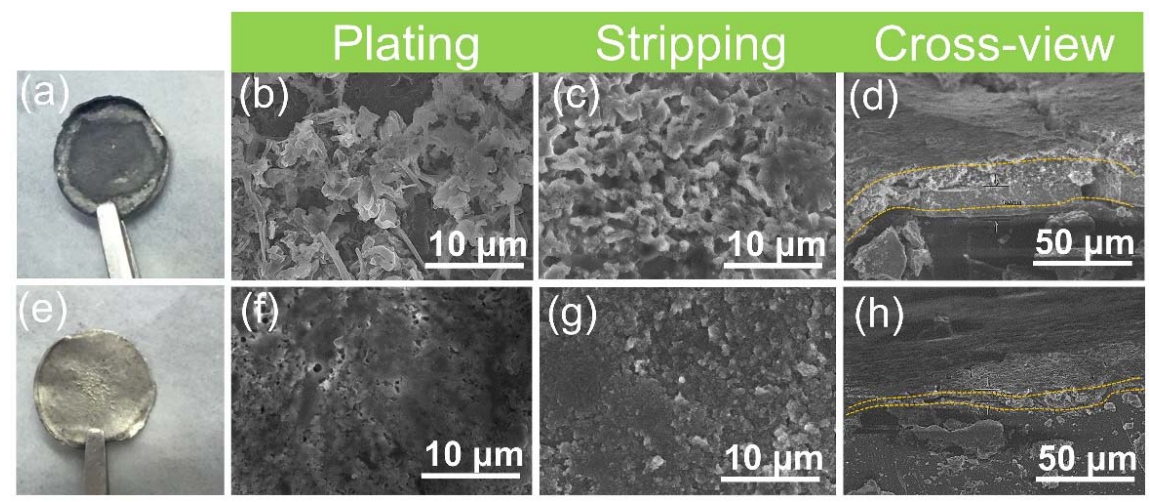

图5 (a, e)循环50圈后 $\mathrm{Li}$ 和 $\mathrm{Li} @ \mathrm{C}$ 电极的光学照片; $(\mathrm{b}, \mathrm{f}) \mathrm{Li}$ 和 $\mathrm{Li} @ \mathrm{C}$ 电极循环50圈后的表面 $\mathrm{SEM}$ 图;

(c, g) Li和 Li@C 电极循环50圈后锂完全溶解状态下的表面SEM图; (d, h) Li和 Li@C电极循环50圈后的截面SEM图

Fig. 5 (a, e) Digital photos of Li and Li@C electrodes after 50 cycles; (b, f) SEM images of Li and Li@C after 50

cycles at the fully plated state; (c, g) SEM images of $\mathrm{Li}$ and Li@C after 50 cycles at the fully stripped state;

(d, h) cross-sectional SEM images of Li and Li@C after 50 cycles.

异质微结构界面层的锂䇴的光学照片。未改性的 锂箔循环后失去了金属光泽, 并转变为灰黑色, $\mathrm{SEM}$ 表征(图5b)显示出表面存在的大量锂枝晶也 印证了这种表观形态上的巨大改变。而具有 $\mathrm{LiC}_{6}$ 异质微结构界面层的锂䇴表面依然保持了金属光 泽(图5e), 同时SEM表征也显示其表面呈现致密平 滑的形态, 未观察到明显的锂枝晶生长, 说明 $\mathrm{LiC}_{6}$ 异质层起到了引导锂离子均匀沉积的作用。图 $5 \mathrm{c}$ 和 $\mathrm{g}$ 分别是未改性锂䈃和 $\mathrm{Li} @ \mathrm{C}$ 电极在循环后锂完 全脱出状态下的表面形貌图, 从中可以看出, 纯锂 䇴表面腐蚀严重, 呈现出不规则的“沟壑”状形貌， 而具有 $\mathrm{LiC}_{6}$ 异质微结构界面层的锂箔在锂溶解状 态下表面仍保持相对的平整性, 说明 $\mathrm{LiC}_{6}$ 异质层
能够改善锂金属锂沉积/溶解的可逆性与均匀性。对 循环后极片截面的形貌表征也可以进一步反映出 有无 $\mathrm{LiC}_{6}$ 异质层的显著性差异。从图 $5 \mathrm{~d}$ 中可以看出 未改性的锂循环后表面形成了厚度超过 $20 \mu \mathrm{m}$ 的 “死锂” 层, 而具有 $\mathrm{LiC}_{6}$ 异质微结构界面层的锂箔的 表面不可逆锂层厚度仅为 $5 \mu \mathrm{m}$ (图 $5 \mathrm{~h}$ )。由此可见, 改性后的锂箔可以实现均匀且致密的锂沉积。

\section{3 全电池性能对比}

为了验证改性后锂䈃在全电池中的实际应用 效果, 我们以磷酸铁锂为正极活性材料, 未改性或 改性后的锂䈋为负极, 组装成扣式全电池进行倍 率性能和长循环性能测试, 结果如图6所示。使用 未改性锂䈃的电池在 $1 C$ 倍率下的初始放电比容量

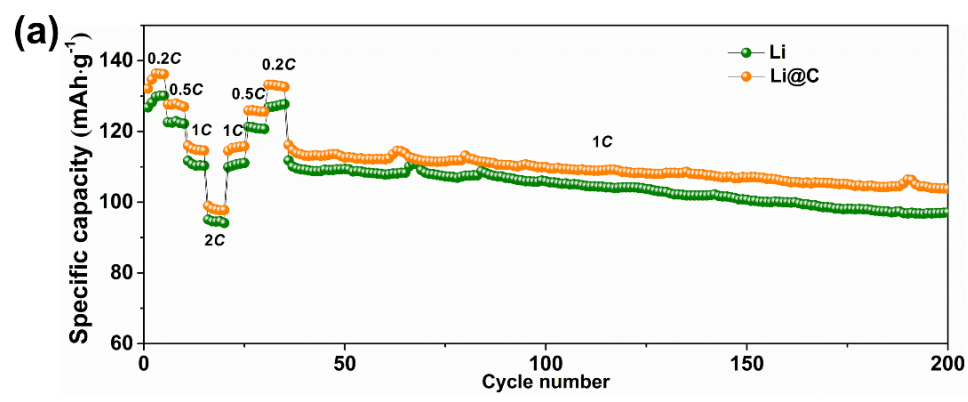

(b)

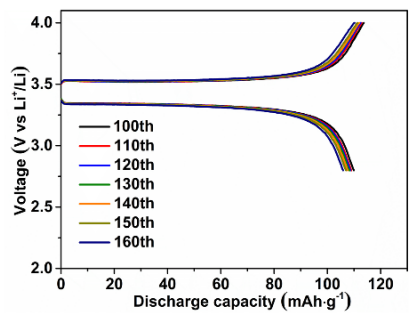

(c)

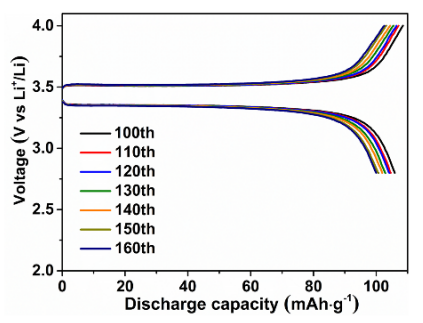

图6 (a) 以纯锂䈃或 $\mathrm{Li} @ \mathrm{C}$ 为负极的扣式全电池的倍率和循环性能; (b-c)电池在不同循环圈数时的充放电曲线

Fig. 6 (a) Rate and cycling performance of coin cells using $\mathrm{Li} @ \mathrm{C}$ or bare $\mathrm{Li}$ as the anode;

(b-c) charge and discharge curves of cells at different cycles. 
为 $110 \mathrm{mAh} \cdot \mathrm{g}^{-1}$, 循环 200 圈后, 比容量降为 95 $\mathrm{mAh} \cdot \mathrm{g}^{-1}$, 容量保持率为 $86 \%$, 而应用改性后锂箔 的电池在 $1 C$ 倍率下具有 $115 \mathrm{mAh} \cdot \mathrm{g}^{-1}$ 的初始放电 比容量, 循环 200 圈后, 容量保持率为 $91 \%$ 。图 $6 \mathrm{~b}$ 和 $6 \mathrm{c}$ 中对于两个电池在不同循环圈数时的充放电 曲线的对比同样发现锂箔表面 $\mathrm{LiC}_{6}$ 异质层改性有 助于提高全电池的循环稳定性。实验中还采用具 有高面载量的LFP极片 $\left(10 \mathrm{mg} \cdot \mathrm{cm}^{-2}\right)$ 进行电化学性 能测试, 结果如图S8所示。对比发现, 在该条件下 使用 $\mathrm{LiC}_{6}$ 异质层改性锂簿的电池同样具有更好的 循环稳定性, 进一步验证了 $\mathrm{LiC}_{6}$ 异质层对于改善 金属锂负极稳定性的作用。

\section{4 结论}

本文以石墨化的碳纳米球为改性材料, 通过 简单的机械辊压方法, 在金属锂表面通过原位固 相反应形成了 $\mathrm{LiC}_{6}$ 异质微结构界面层。该界面层 具有分散电流密度和增强对锂离子吸附作用的特 性, 有助于实现稳定且均匀的锂沉积和溶解。通过 Li-Li对称电池对比发现, 相较于纯锂䈃材, $\mathrm{LiC}_{6}$ 异 质层能够有效抑制锂枝晶的产生, 保持电极与电 解液界面的稳定, 从而大幅提升循环寿命。以磷酸 铁锂为正极的全电池性能测试也表明使用 $\mathrm{LiC}_{6}$ 异 质层改性锂箔负极的电池具有更好的循环稳定 性。研究结果表明, 该简单的表面改性方法在长寿 命锂金属二次电池中的重要应用潜力。

\section{References}

(1) Liu, C.; Jin, Z.; Wang, C.; Liu, H.; Zhang, Q. Energy Chem. 2019, 1 (1), 100003. doi: 10.1016/j.enchem.2019.100003

(2) Peng, Z.; Song, J.; Huai, L.; Jia, H.; Xiao, B.; Zou, L. Adv. Energy Mater. 2019, 9 (42), 1901764. doi: 10.1002/aenm.201901764

(3) Wang, D.; Luan, C.; Zhang, W.; Liu, X.; Wang, P.; Zheng, W. Adv. Energy Mater. 2018, 8 (21), 1800650. doi: 10.1002/aenm.201800650

(4) Cheng, X. B.; Zhang, R.; Zhao, C. Z.; Zhang, Q. Chem. Rev. 2017, 117 (15), 10403. doi: 10.1021/acs.chemrev.7b00115

(5) Yan, C.; Cheng, X. B.; Yao, Y. X.; Shen, X.; Li, B. Q.; Li, W. J.; Zhang, Q. Adv. Mater. 2018, 30 (45), 1804461. doi: 10.1002/adma.201804461

(6) Xu, S. M.; Duan, H.; Shi, J. L.; Zuo, T. T.; Hu, X. C.; Lang, S. Y.; Zhang, X. Nano Res. 2020, 13 (2), 430. doi: $10.1007 / \mathrm{s} 12274-020-2628-\mathrm{Z}$

(7) Li, N. W.; Yin, Y. X.; Yang, C. P.; Guo, Y. G. Adv. Mater. 2016, 28 (9), 1853. doi: 10.1002/adma.201504526

(8) Aurbach, D.; Gofer, Y.; Langzam, J. J. Electrochem. Soc 1989, 136, 11. doi: $10.1149 / 1.2096425$
(9) Gu, Y.; Wang, W. W.; Li, Y. J.; Wu, Q. H.; Tang, S.; Yan, J. W.; Chen, Z. B. Nat. Commun. 2018, 9 (1), 1. doi: $10.1038 / \mathrm{s} 41467-018-03466-8$

(10) Lee, J. I.; Shin, M.; Hong, D. Adv. Energy Mater. 2019, 9 (13), 1803722.1. doi: 10.1002/aenm.201803722

(11) Lee, D.; Sun, S.; Kwon, J. Adv. Mater. 2020, 32, 1905573. doi: 10.1002/adma.201905573

(12) Guo, F.; Wu, C.; Chen, S. ACS. Mater. Lett. 2020, 2 (4), 358. doi: 10.1021/acsmaterialslett.0c00001

(13) Guo, F.; Chen, P.; Kang, T.; Wang, Y. L.; Liu, C. H.; Shen, Y. B.; Lu, W.; Chen, L. W. Acta Phys. -Chim. Sin. 2019, 35 (12), 1365. [ 郭 峰, 陈鹏, 康拓, 王亚龙, 刘承浩, 沈炎宾, 卢威, 陈立桅. 物理化 学学报, 2019, 35 (12), 1365.] doi: 10.3866/PKU.WHXB201903008

(14) Guo, F.; Wang, Y.; Kang, T.; Liu, C.; Shen, Y.; Lu, W.; Chen, L. Energy Storage Mater. 2018, 15, 116. doi: 10.1016/j.ensm.2018.03.018

(15) Shi, P.; Li, T.; Zhang, R.; Shen, X.; Huang, J.; Cheng, X.; Zhang, Q. Adv. Mater. 2019, 31 (8), 1807131. doi: 10.1002/adma.201807131

(16) Cui, J.; Yao, S.; Wu, J.; Kim, J. Adv. Energy Mater. 2019, 9, 1802777. doi: 10.1002/aenm.201802777

(17) Zhao, Q.; Liu, X.; Stalin, S.; Khan, K.; Archer, L. A. Nat Energy. 2019, 4 (5), 365. doi: 10.1038/s41560-019-0349-7

(18) Yan, K.; Lee, H. W.; Gao, T.; Zheng, G.; Yao, H.; Wang, H.; Chu, S. Nano Lett. 2014, 14 (10), 6016. doi: 10.1021/n1503125u

(19) Kim, M. S.; Ryu, J. H.; Lim, Y. R.; Nah, I. W.; Lee, K. R.; Archer, L. A.; Cho, W. I. Nat Energy. 2018, 3 (10), 889. doi: 10.1038/s41560-018-0237-6

(20) Lin, D.; Liu, Y.; Liang, Z.; Lee, H. W.; Sun, J.; Wang, H.; Cui, Y. Nat. Nanotechnol. 2016, 11 (7), 626. doi: 10.1038/NNANO.2016.32

(21) Huang, S.; Tang, L.; Najafabadi, H. S.; Chen, S.; Ren, Z. Nano Energy 2017, 38, 504. doi: 10.1016/j.nanoen.2017.06.030

(22) Zhang, R.; Chen, X. R.; Chen, X.; Cheng, X. B.; Zhang, X. Q.; Yan, C.; Zhang, Q. Angew. Chem. Int. Ed. 2017, 56 (27), 7764. doi: 10.1002/anie.201702099

(23) Shao, Y.; Wang, H.; Gong, Z.; Wang, D.; Zheng, B.; Zhu, J.; Huang, X. ACS Energy Lett. 2018, 3 (6), 1212. doi: 10.1021/acsenergylett.8b00453

(24) Siroma, Z.; Sato, T.; Tamonari, T.; Nagai, R.; Ota, A.; Ioroi, T. J. Power Sources 2016, 316, 215. doi: 10.1016/j.jpowsour.2016.03.059

(25) Salvatierra, R. V.; Yoon, J.; Tour, J. M. Adv. Mater. 2018, 30 (50), 1803869. doi: 10.1002/adma.201803869

(26) Zhou, W. F. Electrochemical measurements. Shanghai Science and Technology Press: Shanghai, 1983; pp. 134-136. [周伟舫. 电化学测 量. 上海: 上海科学技术出版社, 1983: 134-136.] 